\title{
Viscous Fluid Bianchi Type $V$ Cosmological Models with Late Time Acceleration
}

\author{
J. P. Singh, Prashant S. Baghel* and Abhay Singh \\ Department of Mathematical Sciences, A. P. S. University, Rewa - 486003, India \\ Email: jpsinghmp@gmail.com, ps.baghel@yahoo.com, abhaysingh5784@gmail.com
}

\begin{abstract}
A new class of a spatially homogeneous and anisotropic Bianchi type V cosmological models of the universe for viscous fluid distribution within the framework of general relativity is investigated by applying suitable functional form for the Hubble parameter $H$ which yields models of the universe that describe an early deceleration and late time acceleration. We have found that cosmological term $\Lambda$ being very large at initial times relaxes to a genuine cosmological constant at late times. The physical and kinematical parameters of the models are discussed. The models are found to be compatible with the results of recent observations.
\end{abstract}

Keywords: Bianchi V, viscosity, variable cosmological term, anisotropy.

\section{Introduction}

The prediction of standard cosmology that the universe at present is decelerating is contradictory to the recent observational evidences of the high red-shift of type Ia supernovae $[1,2,3,4,5,6]$ and observed data from measurements of the fluctuations in the power spectrum of the cosmic microwave background $[7,8,9,10]$. So within the framework of Einstein's general relativity, the cosmic matter energy should contain, besides baryons, photons, neutrinos and dark matter, another contribution, known as dark energy, which is the source of repulsive gravity. The kinematic effect of dark energy could be equivalent to that of a fluid with negative pressure. Also, at the present era, dark energy dominates over matter and violates the strong energy condition. As the fundamental physical nature of dark energy is unknown, several models for dark energy have been proposed, namely the cosmological constant, quintessence scalar fields, techyonic fluids, generalized forces etc. [11,12,13,14].

The simplest explanation of dark energy is provided by the cosmological constant $\Lambda$, but it needs to be severely fine-tuned due to the problem associated with its energy scale. The vacuum energy density observed today falls below the value of the vacuum energy density predicted by quantum field theory by many order of magnitude [15]. Moreover, matter and radiation energy densities of the expanding universe falls off as $R^{-3}$ and $R^{-4}$ respectively, where $R$ is the scale factor of the universe, while $\Lambda$ remains constant. A possible way out of the trouble is to consider a varying cosmological term especially time dependent, which as long as the universe expands, decays from a huge value at initial times to the small value observed nowadays $[16,17,18,19,20]$.

The distribution of matter can be satisfactorily described by a perfect fluid due to the large scale distribution of galaxies in our universe. However, observed physical phenomena such as the large entropy per baryon and the remarkable degree of isotropy of the cosmic microwave background radiation, suggest the analysis of dissipative effects in cosmology. Furthermore, there are several processes which are expected to give rise to viscous effects. These are the decoupling of neutrinos during the radiation era and the decoupling of radiation and matter during the recombination era. Bulk viscosity is associated with the GUT phase transition and string creation. Weinberg [21] derived general formula for bulk and shear viscosity and used these to evaluate the rate of cosmological entropy production. He deduced that the most general form of the energy momentum tensor allowed by rotational and space-inversion invariance, contains a bulk viscosity term proportional to the volume expansion of model. Misner [22,23] has investigated the effect of viscosity on the evolution of cosmological models. Viscous universe without initial singularity has been studied by Nightingale [24], Heller and Klimek [25]. Roy and Prakash [26] have studied a plane symmetric cosmological models representing a viscous fluid with free gravitational field of non-degenerate Petrov type-I in which coefficient of shear viscosity is constant. Bali and Jain 
$[27,28]$ have studied some expanding and shearing viscous fluid cosmological models in which coefficient of shear viscosity is proportional to the rate of expansion in the model and the free gravitational field is Petrov type D and non-generate. Grøn [29] has reviewed viscous cosmological model and deduced that viscosity plays an important role in the process of isotropization of universe. The effect of bulk viscosity on the cosmological evolution has been investigated by a number of workers in the framework of general theory of relativity $[30,31,32,33,34,35,36]$.

A number of recent observational data suggest that this accelerating phase of the universe is a recent phenomena. So, it is natural to assume that dark energy was insignificant in the early evolution of the universe while it has the dominant contribution at the present accelerating epoch. The transition from a decelerated phase to an accelerated stage of evolution can be due to the domination of dark energy over the other kinds of matter fields. It deserves mention that the same model universe should have decelerating expansion in the early phase of matter era to allow the formation of large structures. To investigate a cosmological scenario with initial deceleration and late time acceleration, authors have proposed several ansatz. Singh [37] studied a cosmological model by assuming a functional relation between Hubble parameter $H$ and average scale factor $R$. Pradhan et al. [38] investigated cosmological models assuming scale factor as an increasing function of cosmic time.

In this work, we assume a relation between Hubble parameter $H$ and cosmic time $t$ as

$$
H=m+n \operatorname{coth} t,
$$

where $\mathrm{m}$ and $\mathrm{n}$ are positive constants. This form of Hubble's parameter $H$ as a function of cosmic time ' $t$ ', we propose is ad hoc in the sense that it is derived from the desired behaviour of the universe, rather than from a known field theory. Berman [39] also studied cosmological models proposing a variation law for the Hubble's parameter without specifying the physics behind such a choice which yields a constant value of decelerating parameter.

\section{Metric and Field Equations}

Bianchi type V space-time in orthogonal form is represented by the line-element

$$
\mathrm{d} s^{2}=-\mathrm{d} t^{2}+A^{2}(t) \mathrm{d} x^{2}+e^{2 \alpha x}\left\{B^{2}(t) \mathrm{d} y^{2}+C^{2}(t) \mathrm{d} z^{2}\right\}
$$

where $\alpha$ is constant. Viscous fluid is represented by the energy momentum tensor

$$
T_{i}{ }^{j}=(\rho+\bar{p}) v_{i} v^{j}+\bar{p} g_{i}{ }^{j}-2 \eta \sigma_{i}{ }^{j},
$$

where $\bar{p}$ is the effective pressure given by

$$
\bar{p}=p-\zeta v_{; i}^{i}
$$

We assume that the cosmic fluid satisfies linear equation of state

$$
p=\omega \rho, \quad 0 \leq \omega \leq 1
$$

Here $\rho$ is matter energy density, $p$, the isotopic pressure, $\eta$ and $\zeta$ are coefficients of shear and bulk viscosity respectively, $v^{i}$ is the flow vector of the fluid satisfying $v_{i} v^{i}=-1$ and $\sigma_{i j}$ is the shear tensor given by

$$
\sigma_{i j}=\frac{1}{2}\left(v_{i ; k} h_{j}^{k}+v_{j ; k} h_{i}^{k}\right)-\frac{1}{3} \theta h_{i j},
$$

where $h_{i j}=g_{i j}+v_{i} v_{j}$ is the projection tensor. Semi-colon (;) stands for covariant derivative. We choose the coordinates to be co-moving, so that

$$
v^{1}=v^{2}=v^{3}=0, v^{4}=1
$$

The Einstein's field equations (in geometrical units $8 \pi G=c=1$ ) with time varying cosmological term $\Lambda(t)$ are given by

$$
R_{i}^{j}-\frac{1}{2} R g_{i}^{j}=-T_{i}^{j}+\Lambda(t) g_{i}^{j}
$$


The field equations for viscous fluid distribution reduce to

$$
\begin{gathered}
p-\left(\zeta-\frac{2}{3} \eta\right) \theta-\Lambda=\frac{\alpha^{2}}{A^{2}}-\frac{\ddot{B}}{B}-\frac{\ddot{C}}{C}-\frac{\dot{B} \dot{C}}{B C}+2 \eta \frac{\dot{A}}{A}, \\
p-\left(\zeta-\frac{2}{3} \eta\right) \theta-\Lambda=\frac{\alpha^{2}}{A^{2}}-\frac{\ddot{C}}{C}-\frac{\ddot{A}}{A}-\frac{\dot{C} \dot{A}}{C A}+2 \eta \frac{\dot{B}}{B}, \\
p-\left(\zeta-\frac{2}{3} \eta\right) \theta-\Lambda=\frac{\alpha^{2}}{A^{2}}-\frac{\ddot{A}}{A}-\frac{\ddot{B}}{B}-\frac{\dot{A} \dot{B}}{A B}+2 \eta \frac{\dot{C}}{C}, \\
\rho+\Lambda=-\frac{3 \alpha^{2}}{A^{2}}+\frac{\dot{A} \dot{B}}{A B}+\frac{\dot{B} \dot{C}}{B C}+\frac{\dot{A} \dot{C}}{A C}, \\
\frac{2 \dot{A}}{A}=\frac{\dot{B}}{B}+\frac{\dot{C}}{C} .
\end{gathered}
$$

From equations (9)-(13), we obtain

$$
\dot{\rho}+(\rho+\bar{p})\left(\frac{\dot{A}}{A}+\frac{\dot{B}}{B}+\frac{\dot{C}}{C}\right)+\dot{\Lambda}=4 \eta \sigma^{2} .
$$

where $\sigma$ is shear scalar given by

$$
\sigma^{2}=\frac{1}{2} \sigma_{i j} \sigma^{i j}
$$

We define the average scale factor $S$ for Bianchi type $V$ space-time as

$$
S^{3}=A B C \text {. }
$$

Generalized Hubble parameter $H$ and generalized deceleration parameter $q$ are defined as

$$
\begin{gathered}
H=\frac{\dot{S}}{S}=\frac{1}{3}\left(H_{1}+H_{2}+H_{3}\right), \\
q=-\frac{\dot{H}}{H^{2}}-1=2-3 \frac{V \ddot{V}}{\dot{V}^{2}},
\end{gathered}
$$

where $H_{1}=\frac{\dot{A}}{A}, H_{2}=\frac{\dot{B}}{B}$ and $H_{3}=\frac{\dot{C}}{C}$ are directional Hubble's factors along $x, y$ and $z$ directions respectively and $V$ is the spatial volume given by

$$
V=S^{3}
$$

Volume expansion $\theta$ and components of shear tensor $\sigma_{i}^{j}$ for the metric (2) are obtained as

$$
\begin{gathered}
\theta=3 H=3 \frac{\dot{S}}{S}, \\
\sigma_{1}^{1}=H_{1}-H, \sigma_{2}^{2}=H_{2}-H, \sigma_{3}^{3}=H_{3}-H, \sigma_{4}^{4}=0 .
\end{gathered}
$$

Shear scalar $\sigma$ is given by

$$
\sigma^{2}=\frac{1}{6}\left[\left(\frac{\dot{A}}{A}-\frac{\dot{B}}{B}\right)^{2}+\left(\frac{\dot{B}}{B}-\frac{\dot{C}}{C}\right)^{2}+\left(\frac{\dot{C}}{C}-\frac{\dot{A}}{A}\right)^{2}\right] .
$$

Equations (10), (11) with the help of (13) give

$$
\frac{\ddot{B}}{B}-\frac{\ddot{C}}{C}+\left(\frac{\dot{B}}{B}-\frac{\dot{C}}{C}\right)\left\{\frac{1}{2}\left(\frac{\dot{B}}{B}+\frac{\dot{C}}{C}\right)+2 \eta\right\}=0 .
$$

On integration, it leads to

$$
\frac{\dot{B}}{B}-\frac{\dot{C}}{C}=\frac{2 k}{S^{3}} e^{-2 \int \eta \mathrm{d} t}
$$


$k$ being constant of integration. From equations (13), (17) and (24), we get

$$
\begin{gathered}
\frac{\dot{A}}{A}=\frac{\dot{S}}{S}, \\
\frac{\dot{B}}{B}=\frac{\dot{S}}{S}+\frac{k}{S^{3}} e^{-2 \int \eta \mathrm{d} t}, \\
\frac{\dot{C}}{C}=\frac{\dot{S}}{S}-\frac{k}{S^{3}} e^{-2 \int \eta \mathrm{d} t} .
\end{gathered}
$$

Equations(9)-(14) can be written in terms of $H, \sigma$ and $q$ as

$$
\begin{gathered}
\bar{p}-\Lambda=(2 q-1) H^{2}-\sigma^{2}+\frac{\alpha}{S^{2}}, \\
\rho+\Lambda=3 H^{2}-\sigma^{2}-3 \frac{\alpha}{S^{2}}, \\
\dot{\rho}+3(\rho+\bar{p}) H+\dot{\Lambda}=4 \eta \sigma^{2} .
\end{gathered}
$$

From equations (28) and (29), we get

$$
\frac{\mathrm{d} \theta}{\mathrm{d} t}=-\theta^{2}+\frac{3}{2}(\rho-p)+\frac{3}{2} \zeta \theta+3 \Lambda+6 \frac{\alpha^{2}}{S^{2}},
$$

which is the Raychaudhuri equation for the given distribution. We observe that a positive $\Lambda$ and bulk viscosity increases the rate of expansion. Shear viscosity does not influence the mean expansion rate. It is also to mention that in the background of Bianchi type $V$ space time the mean expansion rate is higher in comparison to Bianchi type $I$ space time.

For the Zel'dovich fluid $\rho=p$, equation (31) reduces to

$$
\dot{\theta}+\theta^{2}-\frac{3}{2} \zeta \theta-3 \Lambda-6 \frac{\alpha^{2}}{S^{2}}=0,
$$

which is the equation in isotropic case.

\section{Solution and Discussion}

For the assumption (1), scale factor $S$, spatial volume $V$, expansion scalar $\theta$ and deceleration parameter $q$ are obtained as

$$
\begin{gathered}
S=(\sinh t)^{n} e^{m t}, \\
V=(\sinh t)^{3 n} e^{3 m t}, \\
\theta=3(m+n \operatorname{coth} t), \\
q=-1+\frac{n}{(m \sinh t+n \cosh t)^{2}} .
\end{gathered}
$$

We observe that scale factor $S$ is zero at $t=0$ and expansion scalar $\theta$ is infinite at $t=0$, which shows that the universe starts evolving with zero volume at $t=0$ with a big-bang. As $t$ increases, the scale factor $S$ increases whereas volume expansion $\theta$ decreases. At $t=0, q=-1+\frac{1}{n}>0$ provided $0<n<1$ and for $t=\infty, q=-1$. Thus the model universe represents initial decelerating and late time accelerating expansion.

In order to obtain analytical models, we consider specific form of shear viscosity $\eta$ and bulk viscosity $\zeta$ in the following models: 


\subsection{Model I}

We consider that $\eta=\eta_{0}$ and $\zeta=\zeta_{0}$ are constants. For this assumption, equations (25) and (26) become

$$
\begin{gathered}
\frac{\dot{A}}{A}=\frac{\dot{S}}{S}, \\
\frac{\dot{B}}{B}=\frac{\dot{S}}{S}+\frac{k}{S^{3}} e^{-2 \eta_{0} t}, \\
\frac{\dot{C}}{C}=\frac{\dot{S}}{S}-\frac{k}{S^{3}} e^{-2 \eta_{0} t}
\end{gathered}
$$

In this case, shear scalar $\sigma$ comes out to be

$$
\sigma=\frac{k}{S^{3} e^{2 \eta_{0} t}}
$$

Matter density $\rho$, cosmological term $\Lambda$ and shear scalar $\sigma$ for the model are given by

$$
\begin{gathered}
(1+\omega) \rho=2 n \csc h^{2} t-\frac{2 k^{2}}{(\sinh t)^{6 n} e^{\left(6 m+4 \eta_{0}\right) t}} \\
-\frac{2 \alpha^{2}}{(\sinh t)^{2 n} e^{2 m t}}+3(m+n \operatorname{coth} t) \zeta_{0}, \\
\Lambda=3(m+n \operatorname{coth} t)^{2}-\frac{2 n \csc h^{2} t}{(1+\omega)}+\frac{(1-\omega) k^{2}}{(1+\omega)(\sinh t)^{6 n} e^{\left(6 m+4 \eta_{0}\right) t}} \\
-\frac{(1+3 \omega) \alpha^{2}}{(1+\omega)(\sinh t)^{2 n} e^{2 m t}-\frac{3(m+n \operatorname{coth} t)^{2}}{(1+\omega)} \zeta_{0},} \\
\sigma=\frac{k}{(\sinh t)^{3 n} e^{\left(3 m+2 \eta_{0}\right) t}} .
\end{gathered}
$$

We observe that matter density $\rho$, cosmological term $\Lambda$ and shear $\sigma$ all diverge at $t=0$. The model starts with a big-bang from its singular state at $t=0$ and continues to expand till $t=\infty$. In the limit of large times, $\rho \rightarrow \frac{3(m+n) \zeta_{0}}{1+\omega}, \Lambda \rightarrow 3(m+n)^{2}-\frac{3(m+n) \zeta_{0}}{1+\omega}$ and $\sigma \rightarrow 0$. The presence of bulk viscosity is to increase the value of matter density $\rho$ and to decrease the value of vacuum energy density $\Lambda$. We also observe that presence of bulk viscosity prevents the model tending to a de Sitter universe and matter density to become negligible asymptotically. For the model, expansion anisotropy

$$
\frac{\sigma}{\theta}=\frac{k}{3(m+n \operatorname{coth} t)(\sinh t)^{3 n} e^{\left(3 m+2 \eta_{0}\right) t}} .
$$

We observe that $\frac{\sigma}{\theta}$ is infinite at initial epoch and for large values of $t, \frac{\sigma}{\theta} \rightarrow 0$. Therefore, the model approaches isotropy at late times. We notice that the presence of shear viscosity accelerates the process of isotropization.

\subsection{Model II}

We assume

$$
\eta=\frac{1}{\eta_{0}+t}
$$

and

$$
\zeta=\frac{1}{\zeta_{0}+t}
$$

where $\eta_{0}$ and $\zeta_{0}$ are constants [40]. For this assumption, we obtain

$$
\frac{\dot{A}}{A}=\frac{\dot{S}}{S},
$$




$$
\begin{aligned}
& \frac{\dot{B}}{B}=\frac{\dot{S}}{S}+\frac{k}{S^{3}\left(\eta_{0}+t\right)^{2}}, \\
& \frac{\dot{C}}{C}=\frac{\dot{S}}{S}-\frac{k}{S^{3}\left(\eta_{0}+t\right)^{2}}
\end{aligned}
$$

Therefore,

$$
\sigma=\frac{k}{S^{3}\left(\eta_{0}+t\right)^{2}}
$$

Matter density $\rho$, cosmological term $\Lambda$ and shear scalar $\sigma$ for the model take the form

$$
\begin{gathered}
(1+\omega) \rho=2 n \csc h^{2} t-\frac{2 k^{2}}{(\sinh t)^{6 n} e^{6 m t}\left(\eta_{0}+t\right)^{4}} \\
-\frac{2 \alpha^{2}}{(\sinh t)^{2 n} e^{2 m t}}+\frac{3(m+n \operatorname{coth} t)}{\left(\zeta_{0}+t\right)}, \\
\Lambda=3(m+n \operatorname{coth} t)^{2}+\frac{(1-\omega) k^{2}}{(1+\omega)(\sinh t)^{6 n} e^{6 m t}\left(\eta_{0}+t\right)^{4}} \\
-\frac{(1+3 \omega) \alpha^{2}}{(1+\omega)(\sinh t)^{2 n} e^{2 m t}}-\frac{2 n \csc h^{2} t}{(1+\omega)}-\frac{3(m+n \operatorname{coth} t)}{(1+\omega)\left(\eta_{0}+t\right)}, \\
\sigma=\frac{k}{(\sinh t)^{3 n} e^{3 m t}\left(\eta_{0}+t\right)^{2}} .
\end{gathered}
$$

The model starts expanding with a big-bang at $t=0$. At $t=0, \rho, \Lambda$ and $\sigma$ are all infinite but $\eta=\frac{1}{\eta_{0}}$ and $\zeta=\frac{1}{\zeta_{0}}$. For large values of $t, \rho, \eta, \zeta$ and $\sigma$ become zero whereas $\Lambda \rightarrow 3(m+n)^{2}$. We find that cosmological term $\Lambda$ is a decaying function of time and it approaches a small value at late times. This is in agreement with the recent results from supernovae $I a$ observations. We observe that our model tends asymptotically to de Sitter universe with $H=\sqrt{\frac{1}{3}}=m+n$ for the large $t$. Viscous effect in the model vanishes at late times. For the model

$$
\frac{\sigma}{\theta}=\frac{k}{3(m+n \operatorname{coth} t)(\sinh t)^{3 n} e^{3 m t}\left(\eta_{0}+t\right)^{2}} .
$$

For the large values of $t, \frac{\sigma}{\theta} \rightarrow 0$ implying that the model approaches isotropy at late times. It is interesting to note that the $\frac{\sigma}{\theta}$ decreases faster with time due to the presence of shear viscosity. Therefore, one concludes that viscosity plays an important role in the isotropization process of the large scale structure of the universe.

\subsection{Model III}

We assume the form of coefficient of shear viscosity $\eta$ given by Saha [41]

$$
\eta=3 \eta_{0} \frac{\dot{S}}{S}, \quad \eta_{0}=\text { costant }
$$

and coefficient of bulk viscosity $\zeta$ [42] in the form

$$
\zeta=\zeta_{0}+\zeta_{1} \frac{\dot{S}}{S}+\zeta_{2} \frac{\ddot{S}}{S}
$$

$\zeta_{0}, \zeta_{1}$ and $\zeta_{2}$ being constants.

For this choice, equations (25)-(27) become

$$
\begin{gathered}
\frac{\dot{A}}{A}=\frac{\dot{S}}{S}, \\
\frac{\dot{B}}{B}=\frac{\dot{S}}{S}+\frac{k}{S^{3+6 \eta_{0}}},
\end{gathered}
$$




$$
\frac{\dot{C}}{C}=\frac{\dot{S}}{S}-\frac{k}{S^{3+6 \eta_{0}}}
$$

Therefore

$$
\sigma=\frac{k}{S^{3+6 \eta_{0}}}
$$

Shear scalar $\sigma$ and coefficient of shear viscosity $\eta$ for the model are given by

$$
\begin{gathered}
\sigma=\frac{k}{(\sinh t)^{\left(3+6 \eta_{0}\right) n} e^{\left(3+6 \eta_{0}\right) m t}}, \\
\eta=3 \eta_{0}(m+n \operatorname{coth} t) .
\end{gathered}
$$

From equations(35) and (61), we get

$$
\frac{\sigma}{\theta}=\frac{k}{3(m+n \operatorname{coth} t)(\sinh t)^{\left(3+6 \eta_{0}\right) n} e^{\left(3+6 \eta_{0}\right) m t}} .
$$

We observe that the model starts with a big-bang from its singular state at $t=0$ with $\sigma$ and $\eta$ all infinite. In the limit of large times, $\sigma \rightarrow 0$ and $\eta \rightarrow 3 \eta_{0}(m+n)$. Coefficient of shear viscosity tends to a genuine constant for large values of $t$. At $t=0, \frac{\sigma}{\theta}=\infty$ and for $t \rightarrow \infty, \frac{\sigma}{\theta} \rightarrow 0$. Therefore, the model approaches isotropy at late times. The shear viscosity is found to be responsible for faster removal of initial anisotropies in the universe. We discuss the model for different cases of bulk viscosity.

Case 1: $\zeta_{0} \neq 0$ and $\zeta_{1}=\zeta_{2}=0$.

For this choice, we obtain matter density $\rho$, cosmological term $\Lambda$ and coefficient of bulk viscosity $\zeta$ as

$$
\begin{gathered}
(1+\omega) \rho=2 n \csc h^{2} t-\frac{2 k^{2}}{(\sinh t)^{\left(6+12 \eta_{0}\right) n} e^{\left(6+12 \eta_{0}\right) m t}}-\frac{2 \alpha^{2}}{(\sinh t)^{2 n} e^{2 m t}} \\
+3(m+n \operatorname{coth} t) \zeta_{0} . \\
\Lambda=3(m+n \operatorname{coth} t)^{2}-\frac{2 n \csc h^{2} t}{1+\omega}-\frac{(1+3 \omega) \alpha^{2}}{(1+\omega)(\sinh t)^{2 n} e^{2 m t}} \\
+\frac{(1-\omega) k^{2}}{(1+\omega)(\sinh t)^{\left(6+12 \eta_{0}\right) n} e^{\left(6+12 \eta_{0}\right) m t}}-\frac{3(m+n \operatorname{coth} t)}{1+\omega} \zeta_{0} . \\
\zeta=\zeta_{0} .
\end{gathered}
$$

The model has singularity at $t=0$. Matter density $\rho$ and cosmological term $\Lambda$ are infinite at the initial singularity. In the limit of large times (i.e.t $\rightarrow \infty), \rho \rightarrow \frac{3(m+n) \zeta_{0}}{(1+\omega)}$ and $\Lambda \rightarrow 3(m+n)^{2}-\frac{3(m+n) \zeta_{0}}{(1+\omega)}$. Cosmological term $\Lambda$ tends to a genuine constant for large values of $t$. Presence of bulk viscosity prevents the matter density $\rho$ to become negligible for large $t$.

Case 2: $\zeta_{1} \neq 0$ and $\zeta_{0}=\zeta_{2}=0$.

For this assumption, we obtain $\rho, \Lambda$ and $\zeta$ as

$$
\begin{gathered}
(1+\omega) \rho=2 n \csc h^{2} t-\frac{2 k^{2}}{(\sinh t)^{\left(6+12 \eta_{0}\right) n} e^{\left(6+12 \eta_{0}\right) m t}}-\frac{2 \alpha^{2}}{(\sinh t)^{2 n} e^{2 m t}} \\
+3(m+n \operatorname{coth} t)^{2} \zeta_{1}, \\
\Lambda=\frac{3\left(\omega+1-\zeta_{1}\right)(m+n \operatorname{coth} t)^{2}}{1+\omega}-\frac{2 n \csc h^{2} t}{1+\omega}+\frac{(1-\omega) k^{2}}{(1+\omega)(\sinh t)^{\left(6+12 \eta_{0}\right) n} e^{\left(6+12 \eta_{0}\right) m t}} \\
-\frac{(1+3 \omega) \alpha^{2}}{(1+\omega)(\sinh t)^{2 n} e^{2 m t}}, \quad \\
\quad \zeta=\zeta_{1}(m+n \operatorname{coth} t)
\end{gathered}
$$


This model has singularity at $t=0$. It evolves from its singular state at $t=0$. Matter density $\rho$, cosmological term $\Lambda$ and coefficient of bulk $\zeta$ are infinitely large at the beginning of cosmic evolution. In the limit of large times, $\rho \rightarrow \frac{3(m+n)^{2}}{(1+\omega)} \zeta_{1}, \Lambda \rightarrow \frac{3(m+n)^{2}\left(1+\omega-\zeta_{1}\right)}{(1+\omega)}$ and $\zeta \rightarrow \zeta_{1}(m+n)$. The bulk viscosity coefficient being infinitely large at the initial singularity decreases with time. We find that the cosmological term $\Lambda$ is very large at initial time and reduces to a constant at late times. In this case also, matter density attains a constant value at late times on account of presence of bulk viscosity.

Case 3: $\zeta_{0} \neq 0, \zeta_{1} \neq 0$ and $\zeta_{2}=0$.

In this case, we get $\rho, \Lambda$ and $\zeta$ as

$$
\begin{gathered}
(1+\omega) \rho=2 n \csc h^{2} t-\frac{2 \alpha^{2}}{(\sinh t)^{2 n} e^{2 m t}}-\frac{2 k^{2}}{(\sinh t)^{\left(6+12 \eta_{0}\right) n} e^{\left(6+12 \eta_{0}\right) m t}} \\
+3(m+n \operatorname{coth} t)\left[\zeta_{0}+\zeta_{1}(m+n \operatorname{coth} t)\right], \\
\Lambda=\frac{3\left(\omega+1-\zeta_{1}\right)(m+n \operatorname{coth} t)^{2}}{1+\omega}-\frac{2 n \csc h^{2} t}{1+\omega}+\frac{(1-\omega) k^{2}}{(1+\omega)(\sinh t)^{\left(6+12 \eta_{0}\right) n} e^{\left(6+12 \eta_{0}\right) m t}} \\
-\frac{(1+3 \omega) \alpha^{2}}{(1+\omega)(\sinh t)^{2 n} e^{2 m t}}-\frac{3(m+n \operatorname{coth} t)}{1+\omega} \zeta_{0}, \\
\zeta=\zeta_{1}+\zeta_{1}(m+n \operatorname{coth} t) .
\end{gathered}
$$

This model also starts expanding with a big-bang at $t=0$ with $\rho, \Lambda$ and $\zeta$ all infinite. For the large value of $t, \rho \rightarrow \frac{3(m+n)}{(1+\omega)}\left[\zeta_{0}+\zeta_{1}(m+n)\right], \Lambda \rightarrow 3(m+n)^{2}-\frac{3(m+n)}{(1+\omega)}\left[\zeta_{0}+\zeta_{1}(m+n)\right]$ and $\zeta \rightarrow \zeta_{0}+\zeta_{1}(m+n)$. Coefficient of bulk viscosity tends to a genuine constant for large values of $t$. We find that the presence of bulk viscosity is to increase the value of matter density $\rho$ and to decrease the value of vacuum energy density $\Lambda$.

Case 4: $\zeta_{0} \neq 0, \zeta_{1} \neq 0$ and $\zeta_{2} \neq 0$.

For this choice, we obtain $\rho, \Lambda$ and $\zeta$ as

$$
\begin{aligned}
(1+\omega) \rho= & n \csc h^{2} t\left[2-3 \zeta_{2}(m+n \operatorname{coth} t)\right]-\frac{2 \alpha^{2}}{(\sinh t)^{2 n} e^{2 m t}}-\frac{2 k^{2}}{(\sinh t)^{\left(6+12 \eta_{0}\right) n} e^{\left(6+12 \eta_{0}\right) m t}} \\
+ & 3(m+n \operatorname{coth} t)\left[\zeta_{0}+\zeta_{1}(m+n \operatorname{coth} t)+\zeta_{2}(m+n \operatorname{coth} t)^{2}\right], \\
\Lambda= & \frac{3\left(\omega+1-\zeta_{1}\right)(m+n \operatorname{coth} t)^{2}}{1+\omega}+\frac{(1-\omega) k^{2}}{(1+\omega)(\sinh t)^{\left(6+12 \eta_{0}\right) n} e^{\left(6+12 \eta_{0}\right) m t}} \\
& -\frac{n \csc h^{2} t\left[2-3 \zeta_{2}(m+n \operatorname{coth} t)\right]}{1+\omega}-\frac{(1+3 \omega) \alpha^{2}}{(1+\omega)(\sinh t)^{2 n} e^{2 m t}} \\
& -\frac{3(m+n \operatorname{coth} t)\left[\zeta_{0}+\zeta_{2}(m+n \operatorname{coth} t)^{2}\right]}{1+\omega}, \\
& \zeta=\zeta_{0}+(m+n \operatorname{coth} t)\left[\zeta_{1}+\zeta_{2}(m+n \operatorname{coth} t)\right]-\zeta_{2} n \csc h^{2} t .
\end{aligned}
$$

We also observe that the model starts with a big-bang from its singular state $t=0$ with $\rho, \Lambda$ and $\zeta$ all infinite. In the limit of large times(i.e.t $\rightarrow \infty), \rho=\frac{3(m+n)}{(1+\omega)}\left[\zeta_{0}+\zeta_{1}(m+n)+\zeta_{2}(m+n)^{2}\right], \Lambda=$ $\frac{3\left(\omega+1-\zeta_{1}\right)}{1+\omega}(m+n)^{2}-\frac{3(m+n)}{(1+\omega)}\left[\zeta_{0}+\zeta_{2}(m+n)^{2}\right]$ and $\zeta=\zeta_{0}+(m+n)\left[\zeta_{1}+\zeta_{2}(m+n)\right]$. We also find that the cosmological term $\Lambda$ is very large at initial time and relaxes to a small value at late times. In the presence of bulk viscosity, matter density $\rho$ increases and vacuum energy density $\Lambda$ decreases with time. Coefficient of bulk viscosity tends to a genuine constant for large values of $t$. Because of bulk viscosity, matter density does not become negligible and the model does not tend to a de Sitter universe for large values of $t$. 


\subsection{Model IV}

We consider

$$
\eta=3 \eta_{0} \frac{\dot{S}}{S}
$$

and

$$
\zeta=\zeta_{0} \rho,
$$

where $\zeta_{0}$ is constant [43]. In this case, we obtain $\rho, \Lambda$ and $\zeta$ as

$$
\begin{aligned}
& {\left[1+\omega+3 \zeta_{0}(m+n \operatorname{coth} t)\right] \rho=2 n \csc h^{2} t-\frac{2 \alpha^{2}}{(\sinh t)^{2 n} e^{2 m t}}} \\
& -\frac{2 k^{2}}{(\sinh t)^{\left(6+12 \eta_{0}\right) n} e^{\left(6+12 \eta_{0}\right) m t}} \\
& \Lambda=3(m+n \operatorname{coth} t)^{2}-\frac{2 n \csc h^{2} t}{1+\omega+3 \zeta_{0}(m+n \operatorname{coth} t)} \\
& +\left[\frac{1-\omega-3 \zeta_{0}(m+n \operatorname{coth} t)}{1+\omega+3 \zeta_{0}(m+n \operatorname{coth} t)}\right] \frac{k^{2}}{(\sinh t)^{\left(6+12 \eta_{0}\right) n} e^{\left(6+12 \eta_{0}\right) m t}} \\
& -\left[\frac{1+3 \omega+9 \zeta_{0}(m+n \operatorname{coth} t)}{1+\omega+3 \zeta_{0}(m+n \operatorname{coth} t)}\right] \frac{\alpha^{2}}{(\sinh t)^{2 n} e^{2 m t}}, \\
& {\left[\frac{1+\omega+3 \zeta_{0}(m+n \operatorname{coth} t)}{\zeta_{0}}\right] \zeta=2 n \csc h^{2} t-\frac{2 \alpha^{2}}{(\sinh t)^{2 n} e^{2 m t}}} \\
& -\frac{2 k^{2}}{(\sinh t)^{\left(6+12 \eta_{0}\right) n} e^{\left(6+12 \eta_{0}\right) m t}} \text {. }
\end{aligned}
$$

We observe that the model starts with a big-bang from its singular state $t=0$ with $\rho, \Lambda$ and $\zeta$ all infinite. In the limit of large times, $\rho$ and $\zeta$ become zero but $\Lambda \rightarrow 3(m+n)^{2}$. We find that cosmological term $\Lambda$ is a decaying function of time and it approaches a small value at late times. Thus, our model tends asymptotically to a de Sitter universe with $H=\sqrt{\frac{\Lambda}{3}}=m+n$ for large values of $t$. Time evolutions of some cosmological parameters are shown graphically in Figures[1-18].

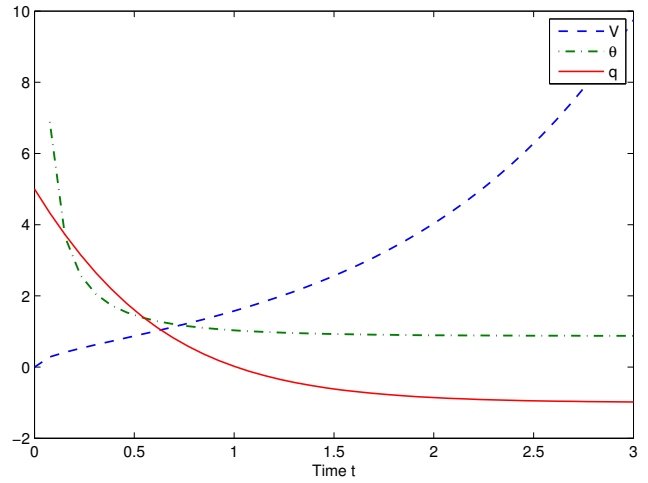

Figure 1. Variation of spatial volume $V$, expansion scalar $\theta$ and deceleration parameter $q$ with cosmic time $t$.

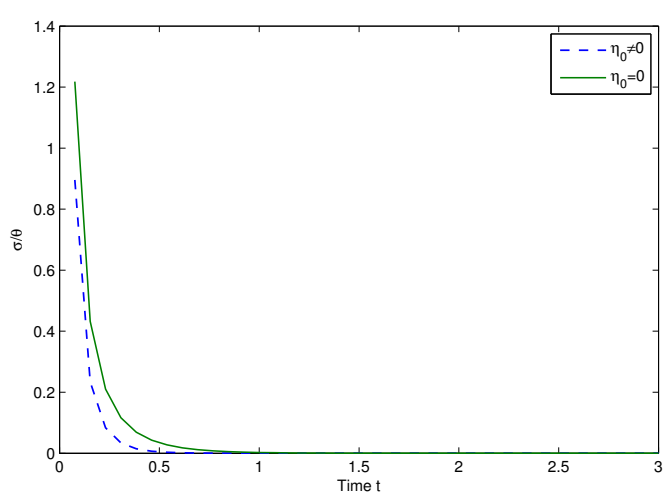

Figure 2. Variation of expansion anisotropy $\frac{\sigma}{\theta}$ with cosmic time $t$ in Model I. 


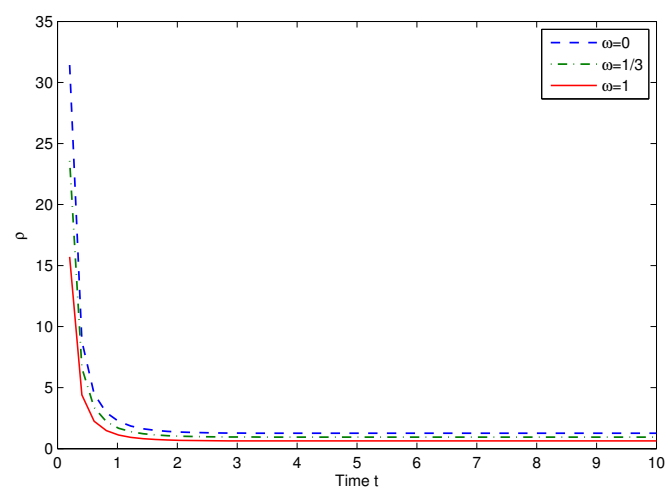

Figure 3. Variation of matter energy density $\rho$ with cosmic time $t$ in Model I.

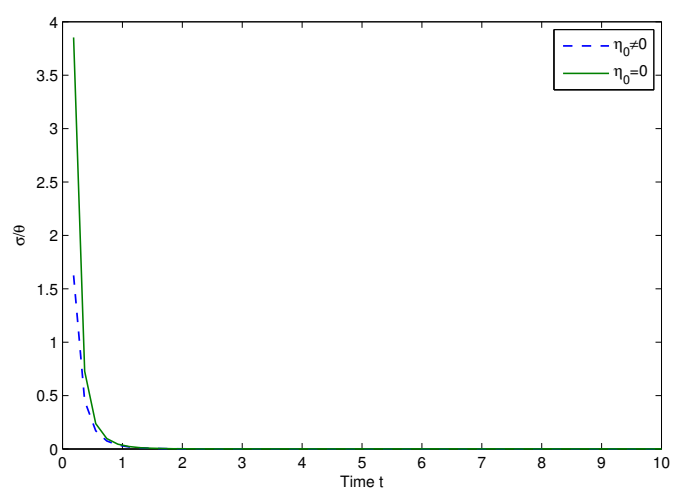

Figure 5. Variation of expansion anisotropy $\frac{\sigma}{\theta}$ with cosmic time $t$ in Model II.

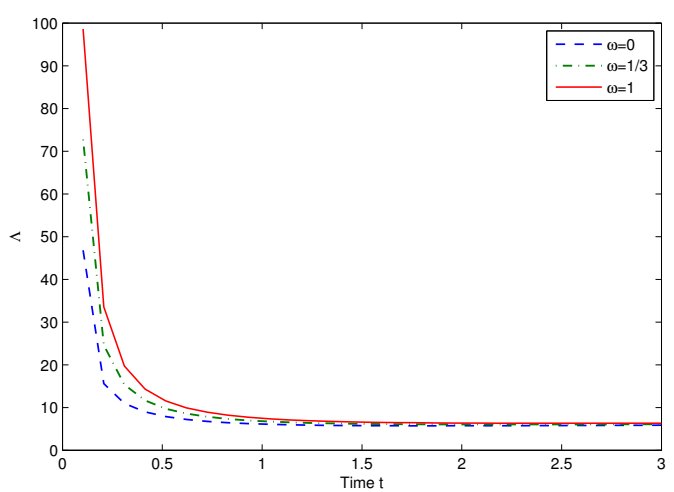

Figure 7. Variation of vacuum energy density $\Lambda$ with cosmic time $t$ in Model II.

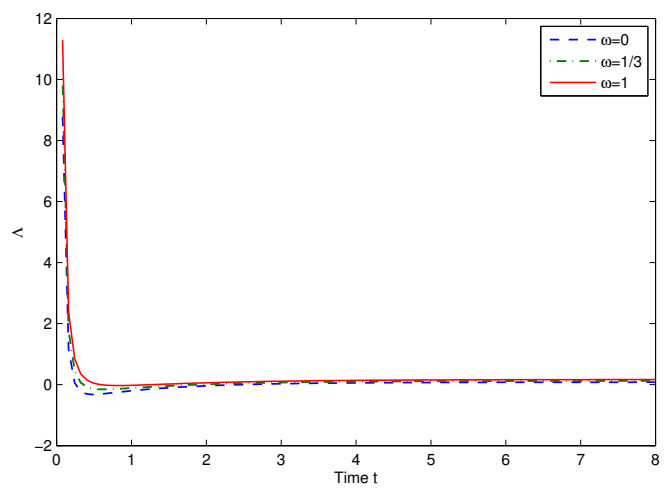

Figure 4. Variation of vacuum energy density $\Lambda$ with cosmic time $t$ in Model I.

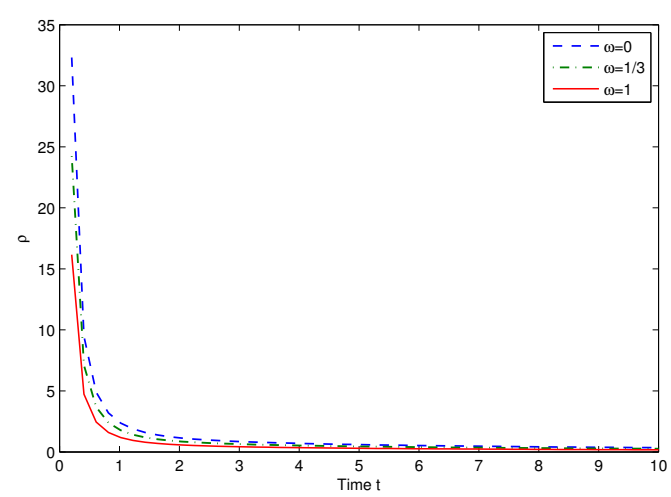

Figure 6. Variation of matter energy density $\rho$ with cosmic time $t$ in Model II.

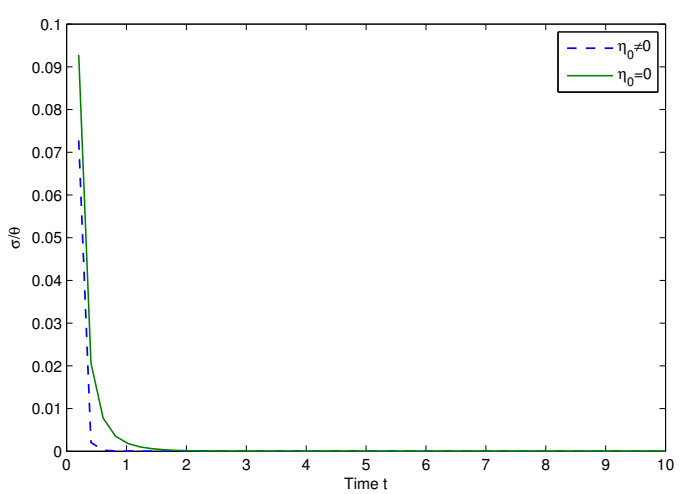

Figure 8. Variation of expansion anisotropy $\frac{\sigma}{\theta}$ with cosmic time $t$ in Model III. 


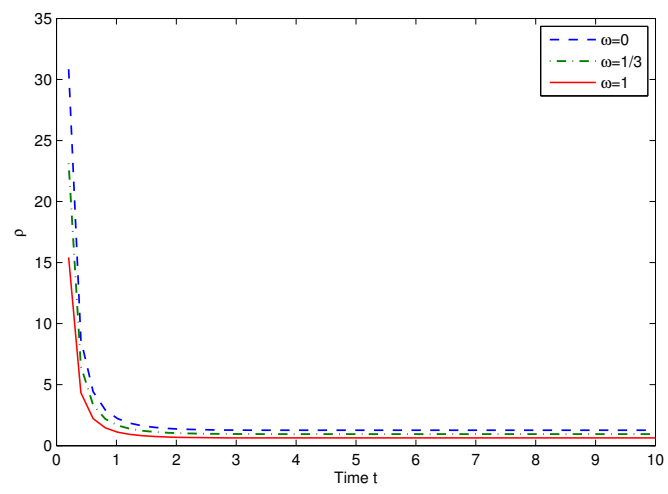

Figure 9. Variation of matter energy density $\rho$ with cosmic time $t$ in model III (case 1).

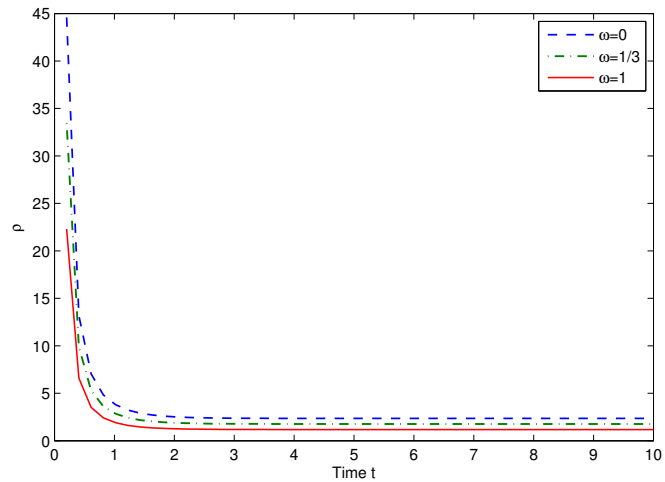

Figure 11. Variation of matter energy density $\rho$ with cosmic time $t$ in model III (case 2).

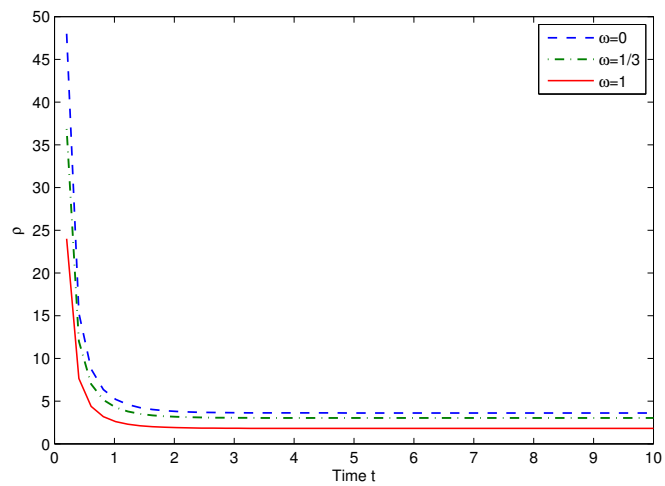

Figure 13. Variation of matter energy density $\rho$ with cosmic time $t$ in model III (case 3 ).

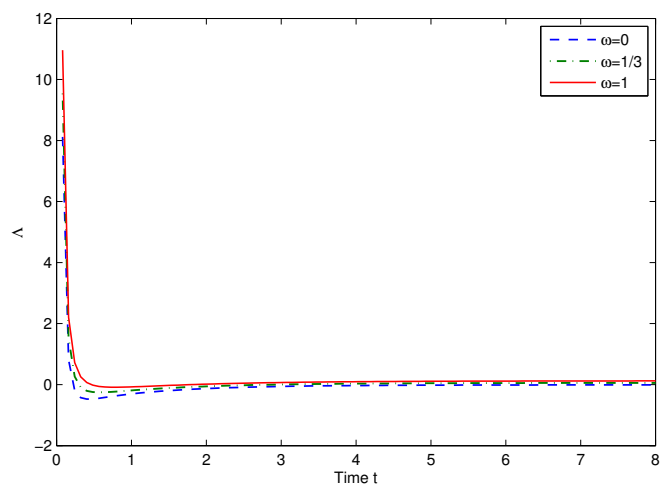

Figure 10. Variation of vacuum energy density $\Lambda$ with cosmic time $t$ in model III ( case 1).

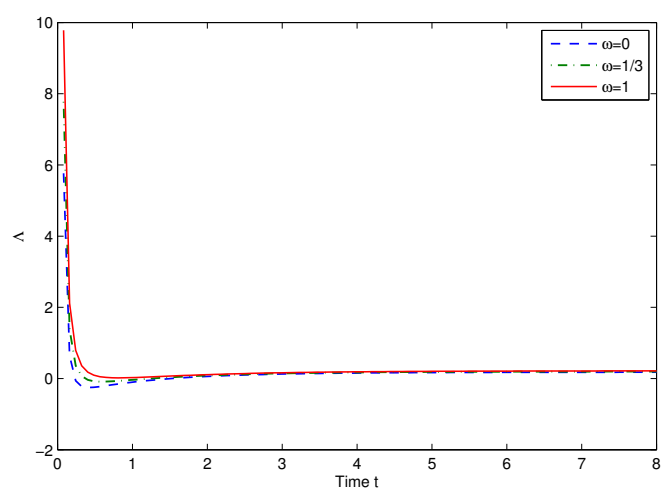

Figure 12. Variation of vacuum energy density $\Lambda$ with cosmic time $t$ in model III ( case 2).

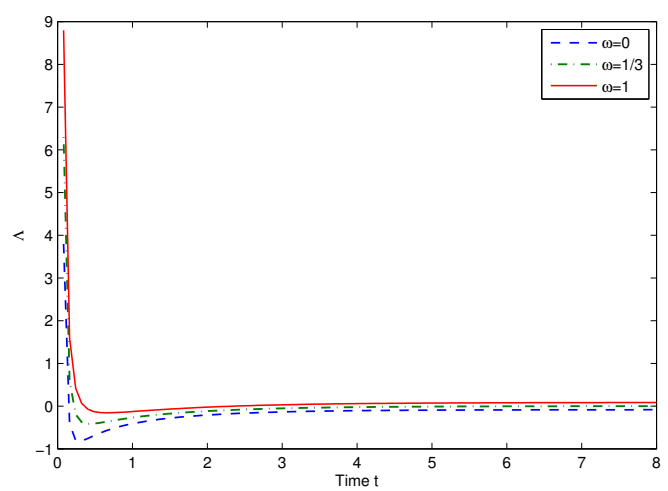

Figure 14. Variation of vacuum energy density $\Lambda$ with cosmic time $t$ in model III ( case 3 ). 


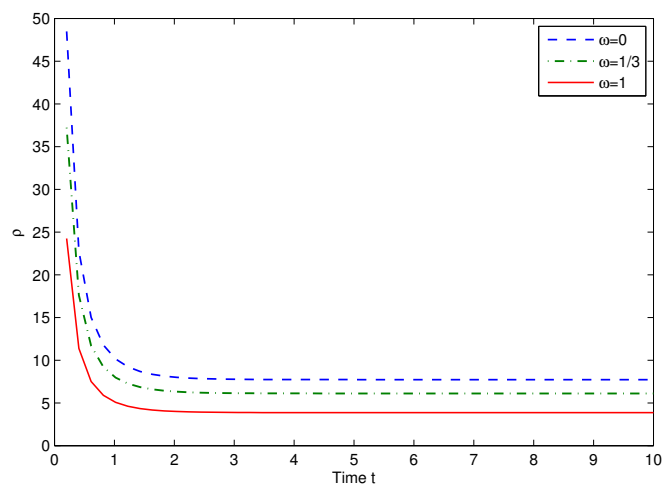

Figure 15. Variation of matter energy density $\rho$ with cosmic time $t$ in model III (case 4).

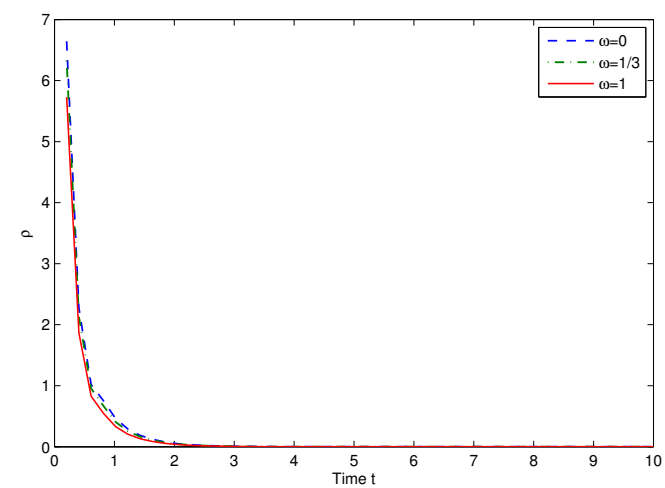

Figure 17. Variation of matter energy density $\rho$ with cosmic time $t$ in model IV.

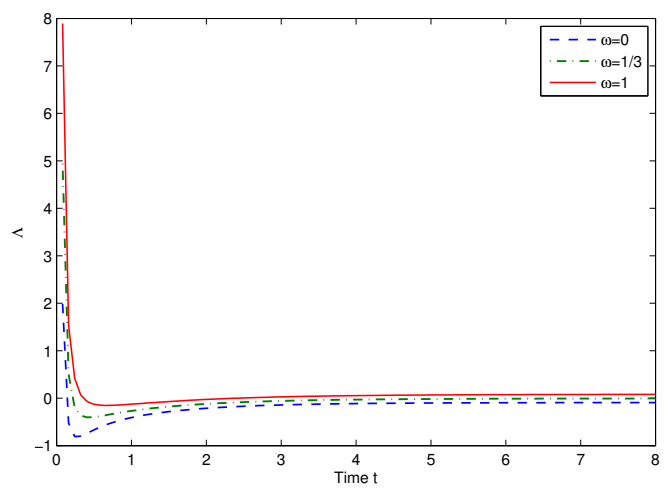

Figure 16. Variation of vacuum energy density $\Lambda$ with cosmic time $t$ in model III ( case 4 ).

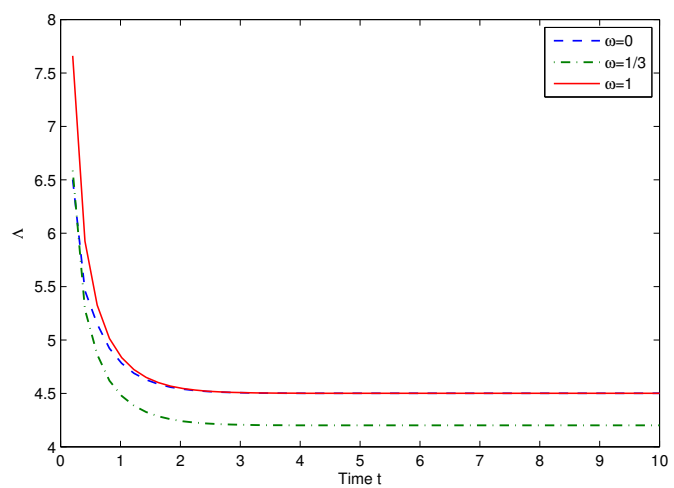

Figure 18. Variation of vacuum energy density $\Lambda$ with cosmic time $t$ in model IV.

\section{Conclusion}

In this paper, we have presented four models of Bianchi type $\mathrm{V}$ cosmological solutions to field equations with viscous fluid in the presence of a cosmological term $\Lambda$ in general relativity. Cosmological models have been obtained by assuming a functional form for Hubble parameter which yields a model of the universe that represents initially decelerating and late time accelerating expansion. The evolution of the universe in such a scenario is shown to be consistent with the present observations predicting an accelerated expansion. We observe that the model approaches isotropy for large values of $t$ and presence of shear viscosity accelerates the isotropization process. Cosmological term $\Lambda$ being very large at initial times relaxes to a genuine cosmological constant asymptotically. The proposed functional form for Hubble parameter $H$ produces cosmological models that give the desired behavior of the universe.

\section{References}

1. A. G. Riess, L. G. Strolger, J. Tonry, S. Casertano, H. C. Ferguson, B. Mobasher, P. Challis, A. V. Filippenko, S. Jha, W. Li, R. Chornock, R. P. Kirshner, B. Leibundgut, M. Dickinson, M. Livio, M. Giavalisco, C. C. Steidel, T. Bentez, and Z. Tsvetanov, "Type Ia supernova discoveries at $z>1$ from the Hubble space telescope: Evidence for past deceleration and constraints on dark energy evolution," Astrophys. J., vol. 607, pp. 665-687, 2004. 
2. A. G. Riess, A. V. Filippenko, P. Challis, A. Clocchiatti, A. Diercks, P. M. Garnavich, R. L. Gilliland, C. J. Hogan, S. Jha, R. P. Kirshner, B. Leibundgut, M. M. Phillips, D. Reiss, B. P. Schmidt, R. A. Schommer, R. C. Smith, J. Spyromilio, C. Stubbs, N. B. Suntzeff, and J. Tonry, "Observational evidence from supernova for an acelerating universe and a cosmological constant," Astron. J., vol. 116, pp. 1009-1038, 1998.

3. S. Perlmutter, G. Aldering, G. Goldhaber, R. A. Knop, P. Nugent, P. G. Castro, S. Deustua, S. Fabbro, A. Goobar, D. E. Groom, I. M. Hook, A. G. Kim, M. Y. Kim, J. C. Lee, N. J. Nunes, R. Pain, C. R. Pennypacker, R. Quimby, C. Lidman, R. S. Ellis, M. Irwin, R. G. McMahon, P. Ruiz-Lapuente, N. Walton, B. Schaefer, B. J. Boyle, A. V. Filippenko, T. Matheson, A. S. Fruchter, N. Panagia, H. J. M. Newberg, and W. J. Couch, "Measurements of $\Omega$ and $\Lambda$ from 42 high-redshift supernovae," Astrophys. J., vol. 517, pp. 565-586, 1999.

4. A. R. Liddle, "Acceleration of the universe," New Astron. Rev., vol. 45, pp. 235-253, 2001.

5. J. L. Tonry, B. P. Schmidt, B. Barris, P. Candia, P. Challis, A. Clocchiatti, A. L. Coil, A. V. Filippenko, P. Garnavich, C. Hogan, S. T. Holland, S. Jha, R. P. Kirshner, K. Krisciunas, B. Leibundgut, W. Li, T. Matheson, M. M. Phillips, A. G. Riess, R. Schommer, R. C. Smith, J. Sollerman, J. Spyromilio, C. W. Stubbs, and N. B. Suntzeff, "Cosmological results from high-z supernovae," Astrophys. J., vol. 594, no. 1, pp. $1-24,2003$.

6. N. Seto, S. Kawamura, and T. Nakamura, "Possibility of direct measurement of the acceleration of the universe using $0.1 \mathrm{~Hz}$ band laser interferometer gravitational wave antenna in space," Phys. Rev. Lett., vol. 87, no. 22, p. 221103, 2001.

7. A. T. Lee, P. Ade, A. Balbi, J. Bock, J. Borrill, A. Boscaleri, P. de Bernardis, P. G. Ferreira, S. Hanany, V. V. Hristov, A. H. Jaffe, P. D. Mauskopf, C. B. Netterfield, E. Pascale, B. Rabii, P. L. Richards, G. F. Smoot, R. Stompor, C. D. Winant, and J. H. P. Wu, "A high spatial resolution analysis of the MAXIMA - 1 cosmic microwave background anisotropy data," Astrophys. J. Lett., vol. 561, no. 1, pp. L1-L6, 2001.

8. R. Stompor, M. Abroe, P. Ade, A. Balbi, D. Barbosa, J. Bock, J. Borrill, A. Boscaleri, P. de Bernardis, P. G. Ferreira, S. Hanany, V. Hristov, A. H. Jaffe, A. T. Lee, E. Pascale, B. Rabii, P. L. Richards, G. F. Smoot, C. D. Winant, and J. H. P. Wu, "Cosmological implications of the MAXIMA - 1 high-resolution cosmic microwave background anisotropy measurement," Astrophys. J. Lett., vol. 561, no. 1, pp. L7-L10, 2001.

9. D. N. Spergel, L. Verde, H. V. Peiris, E. E. Komatsu, M. R. Nolta, C. L. Bennett, M. Halpern, G. Hinshaw, N. Jarosik, A. Kogut, M. Limon, S. S. Meyer, L. Page, G. S. Tucker, J. L. Weiland, E. Wollack, and E. L. Wright, "First-year Wilkinson Microwave Anisotropy Probe (WMAP) observations: Determination of cosmological parameters," Astrophys. J. Suppl., vol. 148, pp. 175-194, 2003.

10. J. L. Sievers, J. R. Bond, J. K. Cartwright, C. R. Contaldi, B. S. Mason, S. T. Myers, S. Padin, T. J. Pearson, U.-L. Pen, D. Pogosyan, S. Prunet, A. C. S. Readhead, M. C. Shepherd, P. S. Udomprasert, L. Bronfman, W. L. Holzapfel, and J. May, "Cosmological parameters from cosmic background imager observations and comparisons with BOOMERANG, DASI, and MAXIMA," Astrophys. J., vol. 591, no. 2, pp. 599-622, 2003.

11. T. Padmanabhan, "Cosmological constant-the weight of the vacuum," Phys. Rep., vol. 380, no. 5, pp. 235-320, 2003.

12. P. J. E. Peebles, "The cosmological constant and dark energy," Rev. Mod. Phys., vol. 75, pp. 559-606, 2003.

13. S. M. Carroll, "The cosmological constant," Living Rev. Relativity, vol. 4, p. 1, 2001.

14. V. Sahni and A. Starobinsky, "The case for a positive cosmological $\Lambda$-term." Int. J. Mod. Phys. D, vol. 9, pp. 373-444, 2000.

15. S. Weinberg, "The cosmological constant problem," Rev. Mod. Phys., vol. 61, pp. 1-24, 1989.

16. O. Bertolami, "Time dependent cosmological term," Nuovo Cim. B, vol. 93, pp. 36-42, 1986.

17. M. Özer and M. O. Taha, "A possible solution to the main cosmological problems," Phys. Lett. B, vol. 171, no. 4, pp. 363-365, 1986.

18. — - "A model of the universe free of cosmological problems," Nucl. Phys. B, vol. 287, pp. 776-796, 1987.

19. K. Freese, F. C. Adams, J. A. Frieman, and E. Mottola, "Cosmology with decaying vacuum energy," Nucl. Phys. B, vol. 287, pp. 797-814.

20. J. P. Singh and R. K. Tiwari, "An LRS Bianchi type-I cosmological model with time-dependent $\Lambda$ term," Int. J. Mod. Phys. D, vol. 16, no. 4, pp. 745-755, 2007.

21. S. Weinberg, "Entropy generation and the survival of protogalaxies in an expanding universe," Astophys. J., vol. 168 , pp. 175-194, 1971.

22. C. W. Misner, "Transport processes in the primordial fireball," Nature, vol. 214, pp. 40-41, 1967.

23. — " "The isotropy of the universe," Astrophys. J., vol. 151, pp. 431-457, 1968.

24. J. D. Nightingale, "Independent investigations concerning bulk viscosity in relativistic homogeneous isotropic cosmologies," Astrophys. J., vol. 185, pp. 105-114, 1973.

25. M. Heller and Z. Klimek, "Viscous universes without initial singularity," Astrophys. Space Sci., vol. 33, no. 2, pp. 37-39, 1975. 
26. S. R. Roy and S. Prakash, "Some solutions of Einstein-Maxwell equations for cylindrically symmetric spacetime with two degrees of freedom in general relativity," Ind. J. Pure Appl. Math., vol. 8, no. 9, pp. 1132-1142, 1977.

27. R. Bali and D. R. Jain, "A gravitationally non-degenerate cosmological model with expanding and shearing viscous fluid in general relativity," Astrophys. Space Sci., vol. 139, no. 1, pp. 175-181, 1987.

28. — - "Some expanding and shearing viscous fluid cosmological models in general relativity," Astrophys. Space Sci., vol. 141, no. 2, pp. 207-216, 1988.

29. Ø. Grøn, "Viscous inflationary universe models," Astrophys. Space Sci., vol. 173, no. 2, pp. 191-225, 1990.

30. A. Pradhan, "Cylindrically symmetric viscous fluid universe in Lyra geometry," J. Math. Phys., vol. 50, no. 2, pp. 022 501-022 501, 2009.

31. - "Plane symmetric viscous fluid universe with decaying vacuum energy density $\Lambda$," FIZIKA $B$, vol. 18 , no. 2, pp. 61-80, 2009.

32. B. Saha, "Bianchi type I universe with viscous fluid," Mod. Phys. Lett. A, vol. 20, pp. 2127-2143, 2005.

33. B. Saha and V. Rikhvitsky, "Anisotropic cosmological models with spinor field and viscous fluid in the presence of a $\Lambda$ term: qualitative solutions," J. Phys. A, vol. 40, no. 46, pp. 14011-14028, 2007.

34. C. P. Singh, S. Kumar, and A. Pradhan, "Early viscous universe with variable gravitational and cosmological 'constants'," Class. Quantum Grav., vol. 24, no. 2, pp. 455-474, 2007.

35. J. P. Singh, "Bulk viscous Bianchi type V cosmological models with decaying cosmological term $\Lambda$," Int. J. Theor. Phys., vol. 49, no. 11, pp. 2734-2744, 2010.

36. R. Bali and P. Kumawat, "Bulk viscous L.R.S. Bianchi type V tilted stiff fluid cosmological model in general relativity," Phys. Lett. B, vol. 665, no. 5, pp. 332-337, 2008.

37. J. P. Singh, "A cosmological model with both deceleration and acceleration," Astrophys. Space Sci., vol. 318, no. 1, pp. 103-107, 2008.

38. A. Pradhan, R. Jaiswal, K. Jotania, and R. K. Khare, "Dark energy models with anisotropic fluid in Bianchi type- $\mathrm{VI}_{0}$ space-time with time dependent deceleration parameter," Astrophys. Space Sci., vol. 337, no. 1, pp. 401-413, 2012.

39. M. S. Berman, "A special law of variation for Hubble's parameter," Nuovo Cimento B, vol. 74, no. 11, pp. 182-186, 1983.

40. A. Pradhan, A. Rai, and S. Otarod, "A new class of Bianchi type I viscous fluid universe with a time dependent cosmological term," FIZIKA B, vol. 17, no. 3, pp. 393-404, 2008.

41. B. Saha, "Bianchi type I universe with viscous fluid," Mod. Phys. Lett. A, vol. 20, no. 28, pp. 2127-2143, 2005.

42. N. Mostafapoor and Ø. Grøn, "Viscous $\Lambda$ CDM universe models," Astrophys. Space Sci., vol. 333, no. 2, pp. 357-368, 2011.

43. R. Maartens, "Dissipative cosmology," Class. Quantum Grav., vol. 12, no. 6, pp. 1455-1466, 1995. 